\title{
Amendment of Calculation Method Airborne Soundproofing of Light Multi-Layer Enclose Structures of Residential Buildings
}

\author{
Jasur Gayratugli Rashidov \\ Senior Lecturer of Department "Building and Structures” at Tashkent Institute of Architecture and Civil \\ Engineering, Tashkent, Uzbekistan
}

Email: jasrash55@gmail.com

\begin{abstract}
This research paper describes airborne soundproofing of multilayer light enclose structures at the stage of designing and reconstruction of residential buildings, with the help of which it is possible to obtain a sufficiently reliable calculation, representing the correct passage and radiation of the sound stream in these structures, taking into account the finiteness of the dimensions, fixing conditions, physical and mechanical characteristics of materials and the method of proper installation.
\end{abstract}

Keywords: Airborne Soundproofing, Frequencies, Multilayer Structures, Residential Buildings.

\section{Introduction}

At present, in the Republic of Uzbekistan, special attention is paid to ensuring the well-being of the people, which is the corresponding goal of implementing the tasks identified in the Strategy of Actions for the further development of the country for 2017-2021 [1]. One of them is the construction of modern buildings. Decree of the President of the Republic of Uzbekistan dated March 13, 2020 No. DP-5963 "On additional measures to deepen reforms in the construction industry of the Republic of Uzbekistan", the National Database of Legislation, as well as other regulatory legal acts adopted in this area, contribute to the achievement of these goals. Today, an urgent task is to ensure the acoustic comfort of the premises, associated with the rational choice of the enclose structures at the design stage.

Construction and installation work must be designed and executed in such a way that the sound perceived and in the vicinity of people is minimized or completely isolated.

Therefore sound level that will not affect their health and will allow them to work in satisfactory conditions. Based on numerous scientific studies, as a result of uncontrolled sound, it can negatively affect human health by the occurrence of various diseases such as: cognitive impairment of the fetus in reproductive people, sleep disturbances, tinnitus, irritation and dysfunction of some human organs (liver, kidneys, etc.). As a result, sound exposure should be considered not only as a source of inconvenience, but also as a source of harm to human health. Sound waves collide with other surfaces, causing them to vibrate and transmit the negative effects of the air around them in adjoining rooms, in which, in terms of sound quality, in many cases this is not enough, since comfort also depends on the sound level of the emitter. Many of these problems are easily resolved at the design stage, while other cases require more costly corrective measures. In particular, one of the main tasks at the design stage is the use of lightweight multi-layer enclosing structures with a relatively small mass that allows to reduce the load and reduce the material consumption of building construction. In this case, it is necessary to ensure the fulfillment of the requirements for the isolation of airborne sound and their expedient distribution of the sound oscillatory processes of the enclosing structures. The rational use of the partitions of sound oscillatory processes 
ensures that the requirements for the strength and stability of structures are met, which makes it possible to increase the speed of installation and reduce its labor intensity.

Sound insulation in the resonant frequency zone of a multilayer structure in strategic buildings may be less than one wall with a total mass of both structures.

\section{Methods of Research}

Researches have shown that with increasing thickness of the air gap and the mass of both plates, the resonant frequency of the mass-elastic-mass system shifts to the low-frequency region. An increase in the sound insulation of multilayer structures is observed at medium and high frequencies lying above the resonance frequency of the mass - elastic - mass system, approximately in an octave.

As the frequency increases from the resonance frequency of the mass - elastic - mass system for multilayer structures with an air gap to the medium and high frequencies, sound insulation takes the form of successively sufficiently high maxima in the zone of ant resonant frequencies of the air gap equal to the sum of sound insulation the first and second structures and narrow minima at the resonant frequencies of the air gap equal to the sound insulation of a single-layer structure with a total mass of both plates. Therefore, in the field of medium and high frequencies, a real multilayer structure has a clear advantage over a single-wall structure of equal mass, i.e. its sound insulation increases due to broad maxima in the region of ant resonant frequencies of the air layer in the controlled zone.

The partition is made of two sheets of a $16 \mathrm{~mm}$ thick slab with a bulk density of $\gamma=680 \mathrm{~kg}$ / $\mathrm{m}^{3}$ along the frame, with an air gap of $50 \mathrm{~mm}$ thick filled with mineral wool slabs, $\gamma=35 \mathrm{~kg} /$ $\mathrm{m}^{3}$ and two gypsum plasterboards of $12.5 \mathrm{~mm}$ thick each with a bulk weight of $\gamma=800 \mathrm{~kg} /$ $\mathrm{m}^{3}$ on a relative of $87 \mathrm{~mm}$ filled with a minelayer $\gamma=48 \mathrm{~kg} / \mathrm{m}^{3}$ with a thickness of $50 \mathrm{~mm}$ (Figure 1)

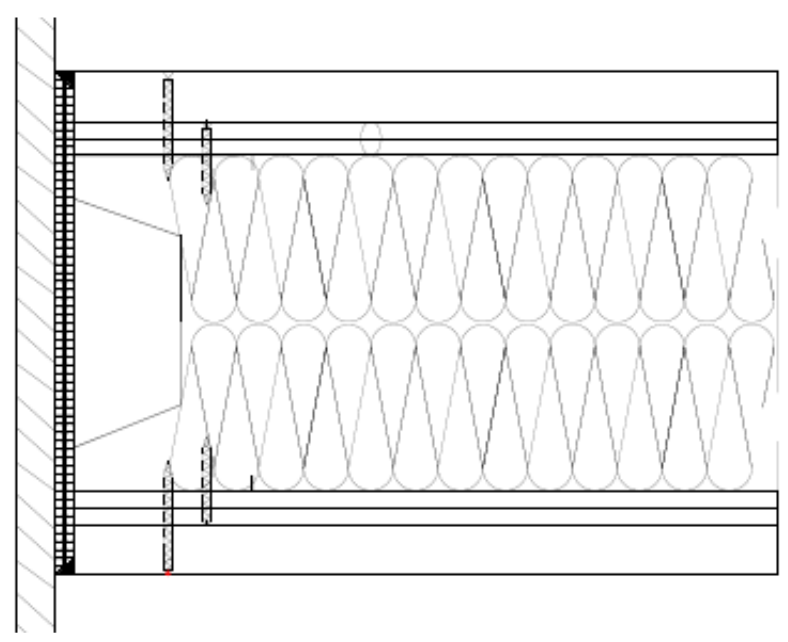

Figure 1. Multi-layer enclose structures acting as partitions of a residential building

The design was used in the construction of inter-apartment partitions at the stage of construction of a residential building in the house on the street. Bakunin, 147, in the city of Penza (Figure 2). 


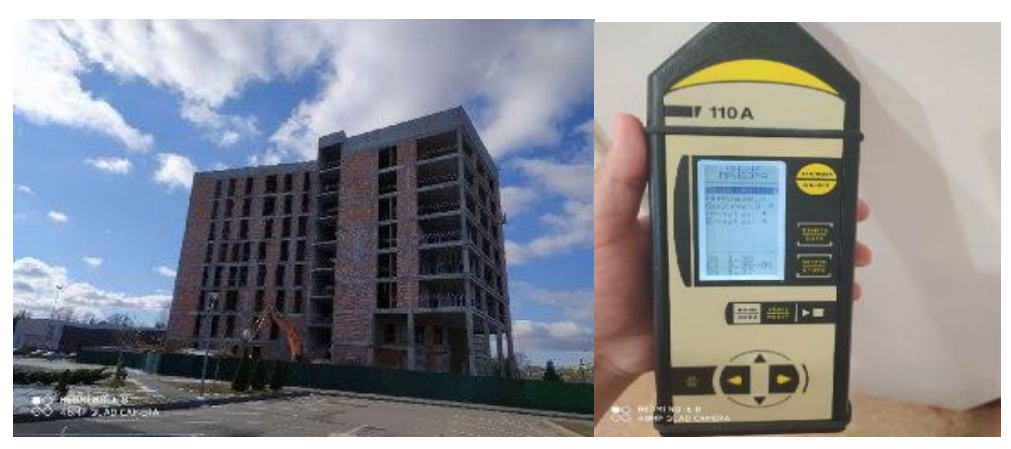

Figure 2. The process of to check soundproofing airborne noise during the construction of a residential building

We build the frequency response of sound insulation for one sheet of drywall in accordance with KMK 2.01.08-20 Noise protection. The coordinates of points B and C are determined by the table.

$$
\begin{gathered}
\mathrm{f}_{\mathrm{B}}=\frac{13500}{16}=843 \approx 800 \mathrm{~Hz} ; \quad \mathrm{R}_{\mathrm{B}}=30,5 \mathrm{~dB} \\
\mathrm{f}_{\mathrm{C}}=\frac{27000}{16}=1687,5 \approx 1600 \mathrm{~Hz} ; \quad \mathrm{R}_{\mathrm{C}}=26 \mathrm{~dB}
\end{gathered}
$$

From point B, draw a segment BA to the left with a slope of $4.5 \mathrm{~dB}$ per octave, and from point $\mathrm{C}$ to the right, draw a segment $\mathrm{CD}$ with a slope of $7.5 \mathrm{~dB}$ per octave.

The broken line $\mathrm{ABCD}$ is the frequency response of airborne noise insulation of one drywall sheet.

We build the auxiliary line A'B'C'D 'by adding the correction $\triangle \mathrm{R} 1$ to increase the surface density to the values of the ABCD line.

In this case, the total surface of the fence includes two sheets of chipboard $16 \mathrm{~mm}$ each, two sheets of gypsum board with a thickness of $12.5 \mathrm{~mm}$ each and mineral wool fillings of $50 \mathrm{~mm}$ $-\gamma=48 \mathrm{~kg} / \mathrm{m}^{3}, 50 \mathrm{~mm}-\gamma=35 \mathrm{~kg} / \mathrm{m}^{3}$

$$
\begin{gathered}
m_{1}=m_{2}=\gamma \mathrm{h}=680 \cdot 0,016=10,88 \mathrm{~kg} / \mathrm{m}^{2} \\
m_{3}=(800 \cdot 0,0125) \cdot 2=20,0 \mathrm{~kg} / \mathrm{m}^{2} \\
m_{4}=(48 \cdot 0,05)+(35 \cdot 0,05)=4,15 \mathrm{~kg} / \mathrm{m}^{2},
\end{gathered}
$$

when,

$$
m_{\text {total }}=(2 \cdot 10,88)+20,0+4,15=45,91 \mathrm{~kg} / \mathrm{m}^{2}
$$




$$
m_{\text {total }} / m_{1}=45,91 / 10,88=4,22 \quad \Delta \mathrm{R}_{1}=9,5 \mathrm{~dB}
$$

Determine the frequency for the plasterboard sheetf $\mathrm{c}^{\prime}$ :

$$
\mathrm{f}_{\mathrm{C}^{\prime}}=38000 / 12,5=3040 \approx 3150 \text { Гц }
$$

We build the auxiliary line $\mathrm{A}^{\prime} \mathrm{B}^{\prime} \mathrm{C}^{\prime} \mathrm{D}$ 'to the frequency $\mathrm{fB}$, adding a correction of $9.5 \mathrm{~dB}$ to the values of the $A B C D$ line (segment $A^{\prime} B^{\prime}$ ), then draw a horizontal segment $B^{\prime} C$ '(point $C^{\prime}$ corresponds to the frequency $\mathrm{f}_{\mathrm{C}^{\prime}}$ ), then we draw a segment $\mathrm{C}^{\prime} \mathrm{D}$ 'with a slope of $7.5 \mathrm{~dB}$ per octave (point D' lies outside the normalized range).

We determine the frequency of the main resonance of the structure by the formula

$$
f_{p}=60 \sqrt{\frac{m_{1}+m_{2}+m_{3}}{d\left(m_{1}+m_{2}\right) \cdot m_{3}}}, H z
$$

where $\mathrm{d}$ - distance between outer covering, $\mathrm{d}=153 \mathrm{~mm}$

$$
f_{p}=60 \sqrt{\frac{10,88+10,88+20,0}{0,153(10,88+10,88) 20,0}}=47,4 \approx 50 \mathrm{~Hz}
$$

At a frequency of $50 \mathrm{~Hz}$, we find the point $\mathrm{F}$ with the ordinate at $(4+2) \mathrm{dB}$ below the auxiliary line A'B'

$$
\mathrm{R}_{\mathrm{f}}=22-4-2=16,0 \mathrm{~dB}
$$

At a frequency of $8 \mathrm{f}_{\mathrm{p}}=400 \mathrm{~Hz}$, we find point $\mathrm{K}$ with ordinate

$$
\mathrm{R}_{\mathrm{K}}=\mathrm{R}_{\mathrm{f}}+\mathrm{H}+2=17,5+27+2=46,5 \mathrm{~dB}
$$

$\mathrm{H}=27 \mathrm{~dB}$.

From point $\mathrm{K}$, we draw a segment KL to a frequency $\mathrm{f}_{\mathrm{B}}=800 \mathrm{~Hz}$ with a slope of $4.5 \mathrm{~dB}$ per octave, $\mathrm{R}_{\mathrm{L}}=49,5 \mathrm{~dB}$.

From point $\mathrm{L}$ to the frequency $\mathrm{f}_{\mathrm{c}^{\prime}}=3150 \mathrm{~Hz}$, we draw a horizontal segment $\mathrm{LM}$, then a segment $\mathrm{MN}$ with a slope of $7.5 \mathrm{~dB}$ per octave.

The broken line FKLMN is a frequency response of airborne noise isolation by a partition with an empty air gap.

At a frequency of $1.6 \mathrm{f}_{\mathrm{p}}=80 \mathrm{~Hz}$, mark the point $\mathrm{Q}$ with ordinate

$$
\mathrm{R}_{\mathrm{Q}}=22,0+5=27,0 \mathrm{~dB}
$$

correction $\Delta \mathrm{R}_{4}=5$ and connect it with point $\mathrm{F}$. 
Next, we construct the frequency response parallel to the FKLMN line by adding the correction $\Delta \mathrm{R}_{4}=5 \mathrm{~dB}$ to its values.

The FQK'L'M'N' broken line represents the frequency response of airborne noise insulation with a given enclosing structure.

In the standardized frequency range of airborne noise insulation is:

Table 1. Frequency range of airborne noise insulation

\begin{tabular}{|c|c|c|c|c|c|c|c|c|}
\hline $\boldsymbol{f}, \boldsymbol{H z}$ & $\mathbf{1 0 0}$ & $\mathbf{1 2 5}$ & $\mathbf{1 6 0}$ & $\mathbf{2 0 0}$ & $\mathbf{2 5 0}$ & $\mathbf{3 1 5}$ & $\mathbf{4 0 0}$ & $\mathbf{5 0 0}$ \\
\hline $\mathbf{R}, \mathbf{d B}$ & 30,0 & 33,5 & 37,0 & 40,0 & 43,5 & 47,0 & 50,0 & 51,5 \\
\hline
\end{tabular}

Continuation of Table 1.

\begin{tabular}{|c|c|c|c|c|c|c|c|c|}
\hline $\boldsymbol{f}, \mathrm{Hz}$ & $\mathbf{6 3 0}$ & $\mathbf{8 0 0}$ & $\mathbf{1 0 0 0}$ & $\mathbf{1 2 5 0}$ & $\mathbf{1 6 0 0}$ & $\mathbf{2 0 0 0}$ & $\mathbf{2 5 0 0}$ & $\mathbf{3 1 5 0}$ \\
\hline $\mathbf{R}, \mathbf{d B}$ & 53,0 & 54,5 & 54,5 & 54,5 & 54,5 & 54,5 & 54,5 & 54,5 \\
\hline
\end{tabular}

To determine the index of airborne noise insulation by the considered design of the interroom partition, we compare the frequency response of sound insulation, (Table 1) with the evaluation curve.

The calculation is carried out in the form of table 2 .

We determine adverse deviations. The sum is $22 \mathrm{~dB}$, which is less than the required $32 \mathrm{~dB}$.

For the value of the insulation index Rw, we take the ordinate of the downwardly estimated curve in the third-octave band with a geometric mean frequency of $500 \mathrm{~Hz}$, i.e. $R_{w}=52 \mathrm{~dB}$.

Table 2. Evaluation curve

\begin{tabular}{|c|l|c|c|c|c|c|c|c|c|}
\hline \multirow{2}{*}{ № } & \multirow{2}{*}{ Parameters } & \multicolumn{6}{|c|}{ The geometric mean frequency of the 1/3-octave band, Hz } \\
\cline { 2 - 10 } & & $\mathbf{1 0 0}$ & $\mathbf{1 2 5}$ & $\mathbf{1 6 0}$ & $\mathbf{2 0 0}$ & $\mathbf{2 5 0}$ & $\mathbf{3 1 5}$ & $\mathbf{4 0 0}$ & $\mathbf{5 0 0}$ \\
\hline 1 & $\begin{array}{l}\text { Estimated frequency } \\
\text { response R, dB }\end{array}$ & 30,0 & 33,5 & 37,0 & 40,0 & 43,5 & 47,0 & 50,0 & 51,5 \\
\hline 2 & EstimatedCurve, dB & 33 & 36 & 39 & 42 & 45 & 48 & 51 & 52 \\
\hline 3 & Adversedeviation, dB & 3,0 & 2,5 & 2,0 & 2,0 & 1,5 & 1,0 & 1,0 & 0,5 \\
\hline 4 & $\begin{array}{l}\text { Airborne sound } \\
\text { insulation index, Rw, } \\
\text { dB }\end{array}$ & & & & & & & & \\
\hline
\end{tabular}

Continuation of Table 2.

\begin{tabular}{|l|l|l|}
\hline № & Parameters & The geometric mean frequency of the 1/3-octave band, $\mathrm{Hz}$ \\
\hline
\end{tabular}




\begin{tabular}{|c|l|c|c|c|c|c|c|c|c|}
\hline & & $\mathbf{6 3 0}$ & $\mathbf{8 0 0}$ & $\mathbf{1 0 0 0}$ & $\mathbf{1 2 5 0}$ & $\mathbf{1 6 0 0}$ & $\mathbf{2 0 0 0}$ & $\mathbf{2 5 0 0}$ & $\mathbf{3 1 5 0}$ \\
\hline 1 & $\begin{array}{l}\text { Estimated frequency } \\
\text { response R, dB }\end{array}$ & 53,0 & 54,5 & 54,5 & 54,5 & 54,5 & 54,5 & 54,5 & 54,5 \\
\hline 2 & EstimatedCurve, dB & 53 & 54 & 55 & 56 & 56 & 56 & 56 & 56 \\
\hline 3 & Adversedeviation, dB & - & - & 0,5 & 1,5 & 1,5 & 1,5 & 1,5 & 1,5 \\
\hline 4 & $\begin{array}{l}\text { Airborne sound } \\
\text { insulation index, Rw, } \\
\text { dB }\end{array}$ & & & & & & & \\
\hline
\end{tabular}

\section{Conclusion}

Consequently, the sound insulation of lightweight building envelopes mainly depends on the physic mechanical characteristics of the materials used, the design of the enclosures, the conditions of connection with adjacent enclosing elements, etc. In this regard, the Anteroom Partition Structure has an air noise insulation index of $\mathrm{R}_{\mathrm{w}}=52 \mathrm{~dB}$. The anteroom partition meets the regulatory requirements for airborne sound insulation $R_{w}=R_{w}^{H}=52 d B$

\section{References:}

Pirmatov, RakhmatillaKhamidullaevich, and JasurGayratUgliRashidov. "Research Of The Acoustic Parameters Of Halls And Practical Methods Of Eliminating Acoustic Defects." The American Journal of Engineering and Technology 2.12 (2020): 7-13.

KMK 2.01.08-20 Noise protection. Tashkent, 2019, 100 p.

Rashidov J. Ensuring acoustic comfort from airborne noise of office buildings in the Republic of Uzbekistan. Architecture. Construction. Design. TIACE. Tashkent, No. 3 2019, 152-155 pp.

Rashidov, Jasur. "Measuring sound insulation of air noise." Theoretical \& Applied Science 12 (2019): 121-123.

Rashidov J. "Isolation of airborne noise of building envelopes using the example of building a residential complex" / collection I Republican Scientific and Practical Conference of Young Scientists.Tashkent, TIACE. 02.27.2019, 79-82 pp.

PirmatovR.Kh., Zakharov A. V., Rashidov J.G. Graphical method for calculating sound insulation of air noise of single layer enclosing structures. International Journal of Advanced Research in Science, Engineering and Technology. India. 6/7/2019, 10294-10298 p.

RashidovJ. Effective ways to reduce the noise of building envelopes in the Republic of Uzbekistan. Special issue of the journal Architecture. Construction. Design. TIACE. Tashkent, No. 2 2019, 83-86 pp.

Rashidov J. Determination of noise insulation through two-layer drywall. Architecture. Construction. Design. TIACE.Tashkent, № 1-2 2019,115-118 pp. 
PirmatovR.Kh., Zakharov A.V. About the dependence of sound transmission on the angle of incidence on the boundary of media or a massive layer // Journal of Problems of Mechanics. 2018. No. 1. p. 50-55. 105.

Rashidov, J. "Sound-insulation technology for ventilated facades, The most urgent issues of the city building and its convergence." Collection of scientific works on the results of Republican scientific-technical conference. Tashkent. 2017.

Zhou, J. Sound transmission through a double-panel construction lined with poroelastic material in the presence of mean flow / J. Zhou, A. Bhaskar, X. Zhang // Journal of Sound and Vibration. - 2013. - 332 (16) . - P. 3724-3734.

Viochek, S. P. Sound transmission through multiple structures // IASA. - 2012. - Vol.21, №.4. - P. 419-428. 